\title{
A LOCALLY ANISOTROPIC REGULARITY CRITERION FOR THE NAVIER-STOKES EQUATION IN TERMS OF VORTICITY
}

\author{
EVAN MILLER
}

(Communicated by Catherine Sulem)

\begin{abstract}
In this paper, we will prove a regularity criterion that guarantees solutions of the Navier-Stokes equation must remain smooth so long as the vorticity restricted to a plane remains bounded in the scale critical space $L_{t}^{4} L_{x}^{2}$, where the plane may vary in space and time as long as the gradient of the unit vector orthogonal to the plane remains bounded. This extends previous work by Chae and Choe that guaranteed that solutions of the Navier-Stokes equation must remain smooth as long as the vorticity restricted to a fixed plane remains bounded in a family of scale critical spaces. This regularity criterion also can be seen as interpolating between Chae and Choe's regularity criterion in terms of two vorticity components and Beirão da Veiga and Berselli's regularity criterion in terms of the gradient of vorticity direction. In physical terms, this regularity criterion is consistent with key aspects of the Kolmogorov theory of turbulence, because it requires that finite-time blowup for solutions of the Navier-Stokes equation must be fully three dimensional at all length scales.
\end{abstract}

\section{INTRODUCTION}

The Navier-Stokes equation is the fundamental equation of fluid mechanics. The incompressible Navier-Stokes equation is given by

$$
\begin{array}{r}
\partial_{t} u-\Delta u+(u \cdot \nabla) u+\nabla p=0 \\
\nabla \cdot u=0,
\end{array}
$$

where $p$ is determined entirely by $u$ by convolution with the Poisson kernel,

$$
p=(-\Delta)^{-1} \sum_{i, j=1}^{3} \frac{\partial u_{j}}{\partial x_{i}} \frac{\partial u_{i}}{\partial x_{j}} .
$$

The Navier-Stokes equation is best viewed as an evolution equation on the space of divergence free vector fields rather than as a system of equations, and this is the vantage point we will adopt in this paper.

Two other fundamentally important objects for the study of the Navier-Stokes equation are the strain and the vorticity. The strain is the symmetric part of $\nabla u$, and is given by

$$
S_{i j}=\frac{1}{2}\left(\partial_{i} u_{j}+\partial_{j} u_{i}\right)
$$

Received by the editors July 29, 2020.

2020 Mathematics Subject Classification. Primary 35Q30. 
The vorticity is the curl of the velocity, $\omega=\nabla \times u$. It is a vector representation of the anti-symmetric party of $\nabla u$, with

$$
A=\frac{1}{2}\left(\begin{array}{ccc}
0 & \omega_{3} & -\omega_{2} \\
-\omega_{3} & 0 & \omega_{1} \\
\omega_{2} & -\omega_{1} & 0
\end{array}\right)
$$

where

$$
A_{i j}=\frac{1}{2}\left(\partial_{i} u_{j}-\partial_{j} u_{i}\right) .
$$

Note that this representation implies that for all $v \in \mathbb{R}^{3}$,

$$
A v=\frac{1}{2} v \times \omega .
$$

The evolution equation for the strain is given by

$$
\partial_{t} S-\Delta S+(u \cdot \nabla) S+S^{2}+\frac{1}{4} \omega \otimes \omega-\frac{1}{4}|\omega|^{2} I_{3}+\operatorname{Hess}(p)=0,
$$

and the evolution equation for vorticity is given by

$$
\partial_{t} \omega-\Delta \omega+(u \cdot \nabla) \omega-S \omega=0 .
$$

Before we proceed, we should define a number of spaces. We will take $\dot{H}^{1}\left(\mathbb{R}^{3}\right)$ to be the homogeneous Hilbert space with norm

$$
\|u\|_{\dot{H}^{1}}^{2}=\int_{\mathbb{R}^{3}} 4 \pi^{2}|\xi|^{2}|\hat{u}(\xi)|^{2} \mathrm{~d} \xi .
$$

We will note that for all $u \in \dot{H}^{1}$, we have

$$
\|u\|_{\dot{H}^{1}}^{2}=\|\nabla u\|_{L^{2}}^{2} .
$$

We will take the inhomogeneous Hilbert space $H^{1}\left(\mathbb{R}^{3}\right)$ to be the space with norm

$$
\|u\|_{H^{1}}^{2}=\|u\|_{L^{2}}^{2}+\|u\|_{\dot{H}^{1}}^{2}
$$

Finally we will take the mixed Lebesgue space $L_{t}^{p} L_{x}^{q}$ to be the Banach space

$$
L_{t}^{p} L_{x}^{q}=L^{p}\left([0, t) ; L^{q}\left(\mathbb{R}^{3}\right)\right) .
$$

We will note in particular that

$$
\|u\|_{L_{t}^{\infty} L_{x}^{\infty}}=\operatorname{essip}_{(x, \tau) \in \mathbb{R}^{3} \times[0, t)}|u(x, \tau)|
$$

In his foundational work on the Navier-Stokes equation, Leray proved the global existence of weak solutions to the Navier-Stokes equation satisfying an energy inequality for arbitrary initial data $u^{0} \in L^{2}$ [15. Such solutions, however, are not known to be either smooth or unique. Kato and Fujita introduced the notion of mild solutions [7], which are solutions that satisfy the Navier-Stokes equation,

$$
\partial_{t} u-\Delta u=-(u \cdot \nabla) u-\nabla p,
$$

in the sense of convolution with the heat kernel as in Duhamel's formula. They used this notion of solution, in particular the higher regularity that can be extracted from the heat kernel, to show that the Navier-Stokes equation has unique, smooth solutions locally in time for arbitrary initial data $u^{0} \in \dot{H}^{1}$. Mild solutions are only known to exist locally in time, however, and so this approach based on the heat semigroup cannot guarantee the existence of global-in-time smooth solutions of the Navier-Stokes equation. It is one of the biggest open questions in the field 
of nonlinear PDEs whether smooth solutions of the Navier-Stokes equations can develop singularities in finite time. If the $\dot{H}^{1}$ norm remains bounded this guarantees that a solution of the Navier-Stokes equation must remain smooth, but there is no known bound on the growth of the $\dot{H}^{1}$ norm of $u$. One bound we do have for smooth solutions of the Navier-Stokes equation is the energy equality.

Proposition 1.1. Suppose $u \in C\left(\left[0, T_{\max }\right) ; H^{1}\left(\mathbb{R}^{3}\right)\right)$ is a mild solution to the Navier-Stokes equation. Then for all $0<t<T_{\max }$

$$
\frac{1}{2}\|u(\cdot, t)\|_{L^{2}}^{2}+\int_{0}^{t}\|\nabla u(\cdot, \tau)\|_{L^{2}}^{2} \mathrm{~d} \tau=\frac{1}{2}\left\|u^{0}\right\|_{L^{2}}^{2} .
$$

The energy equality gives us bounds on solutions $u$ of the Navier-Stokes equation in $L_{t}^{\infty} L_{x}^{2}$ and $L_{t}^{2} \dot{H}_{x}^{1}$, but this cannot guarantee regularity, because both of these bounds are supercritical with respect to the scale invariance of the Navier-Stokes equation. The solution set of the Navier-Stokes equation is invariant under the rescaling

$$
u^{\lambda}(x, t)=\lambda u\left(\lambda x, \lambda^{2}, t\right)
$$

If we do have a bound on $u$ in a scale invariant $L_{t}^{p} L_{x}^{q}$ space then that is enough to guarantee regularity. In particular, the Ladyzhenskaya-Prodi-Serrin regularity criterion [13, 23, 24] states that if a smooth solution of the Navier-Stokes equation develops a singularity in finite-time $T_{\max }<+\infty$, then for all $\frac{2}{p}+\frac{3}{q}=1,3<q \leq+\infty$, then

$$
\int_{0}^{T_{\max }}\|u(\cdot, t)\|_{L^{q}}^{p} \mathrm{~d} t=+\infty .
$$

It is easy to check that this regularity criterion is scale critical with respect to the rescaling in (1.16). This result was extended to the endpoint case, $p=+\infty, q=3$, by Escauriaza, Seregin, and Šverák [6], where they proved that if $T_{\max }<+\infty$, then

$$
\limsup _{t \rightarrow T_{\max }}\|u(\cdot, t)\|_{L^{3}\left(\mathbb{R}^{3}\right)}=+\infty .
$$

There have been many extensions of these regularity criteria, far more than we can discuss here, so we will confine ourselves to discussing regularity criteria directly related to this paper. For a very thorough overview of the literature on regularity criteria for solutions to the Navier-Stokes equation, see Chapter 11 in [14].

One regularity criterion proven by Chae and Choe [2] has a particular geometric significance. Chae and Choe prove a scale critical regularity criterion on two components of vorticity. For a two dimensional solution of the Navier-Stokes equation in the $x y$ plane, the vorticity is entirely in the $z$ direction, perpendicular to the $x y$ plane. This means that if the vorticity restricted to the $x y$ plane or, using rotational invariance, any fixed plane, remains bounded in a scale critical space, then the solution is not too far from being two dimensional. The size of the vorticity restricted to a plane can be interpreted as a measure of how fully three dimensional a solution of the Navier-Stokes equation is, so Chae and Choe's regularity criterion can be interpreted as saying that blowup must be fully three dimensional. Chae and Choe's result is the following. 
Theorem 1.2. Suppose $u \in C\left(\left[0, T_{\max }\right) ; \dot{H}^{1}\left(\mathbb{R}^{3}\right)\right)$ is a mild solution of the Navier-Stokes equation, and let $v \in \mathbb{R}^{3},|v|=1$. Then for all $\frac{3}{q}+\frac{2}{p}=2, \frac{3}{2}<$ $q<+\infty$, there exists $C_{q}>0$ depending only on $q$ such that for all $0<t<T_{\max }$

$$
\|u(t)\|_{\dot{H}^{1}}^{2} \leq\|u(0)\|_{\dot{H}^{1}}^{2} \exp \left(C_{q} \int_{0}^{t}\|v \times \omega(\cdot, \tau)\|_{L^{q}}^{p} \mathrm{~d} \tau\right) .
$$

In particular, if $T_{\max }<+\infty$, then

$$
\int_{0}^{T_{\max }}\|v \times \omega(\cdot, t)\|_{L^{q}}^{p} \mathrm{~d} t=+\infty .
$$

Remark 1.3. Chae and Choe's result is not stated in this form in [2]. Chae and Choe prove a regularity criterion on two components of the vorticity, $\left(\omega_{1}, \omega_{2}, 0\right)$. However, we can see that

$$
e_{3} \times \omega=\left(-\omega_{2}, \omega_{1}, 0\right) .
$$

This means that a regularity criterion on $\left(\omega_{1}, \omega_{2}, 0\right)$ is equivalent to a regularity criterion on $e_{3} \times \omega$. Using the rotational invariance of the Navier-Stokes equation, this is equivalent to a regularity criterion on $v \times \omega$ for any fixed unit vector $v \in \mathbb{R}^{3}$. We note that the Navier-Stokes equation is rotationally invariant in the sense that if $Q \in S O(3)$ is a rotation matrix on $\mathbb{R}^{3}$, and $u$ is a solution of the Navier-Stokes equation, then $u^{Q}$ is also a solution of the Navier-Stokes equation where

$$
u^{Q}(x, t)=Q^{t r} u(Q x, t) .
$$

See chapter 1 in [16] for further discussion.

We will note that because $\omega=\nabla \times u$ is a derivative of $u$, the vorticity has the rescaling,

$$
\omega^{\lambda}(x, t)=\lambda^{2} \omega\left(\lambda x, \lambda^{2} t\right) .
$$

If $\omega$ is a solution of the vorticity equation, then so is $\omega^{\lambda}$ for all $\lambda>0$. Theorem 1.2 is critical with respect to this rescaling.

Theorem 1.2 was then extended into Besov spaces by Chen and Zhang 25] and more recently into the endpoint Besov space, $\dot{B}_{\infty, \infty}^{-\frac{3}{q}}$, for $3<q<+\infty$ by Guo, Kučera, and Skalák [8]. This is an improvement on Theorem 1.2, because we have an embedding $L^{q} \hookrightarrow \dot{B}_{\infty, \infty}^{-\frac{3}{q}}$, and $\dot{B}_{\infty, \infty}^{-\frac{3}{q}}$ is the largest translation invariant Banach space with the same scaling relation as $L^{q}$. This is therefore, the furthest advance that can be made to Chae and Choe's regularity criterion solely by loosening the assumptions on the space.

Another regularity criterion with geometric significance is the regularity criterion in terms of the positive part of the intermediate eigenvalue of the strain matrix. This was first proven by Neustupa and Penel in [19 21] and independently by the author using different methods in [17].

Theorem 1.4. Suppose $u \in C\left(\left[0, T_{\max }\right) ; \dot{H}^{1}\left(\mathbb{R}^{3}\right)\right)$ is a mild solution of the Navier-Stokes equation. Let $\lambda_{1}(x, t) \leq \lambda_{2}(x, t) \leq \lambda_{3}(x, t)$ be the eigenvalues of $S(x, t)$, and let $\lambda_{2}^{+}(x, t)=\max \left\{0, \lambda_{2}(x, t)\right\}$. Then for all $\frac{3}{q}+\frac{2}{p}=2, \frac{3}{2}<q \leq+\infty$, there exists $C_{q}>0$ depending only on $q$ such that for all $0<t<T_{\max }$

$$
\|u(t)\|_{\dot{H}^{1}}^{2} \leq\|u(0)\|_{\dot{H}^{1}}^{2} \exp \left(C_{q} \int_{0}^{t}\left\|\lambda_{2}^{+}(\cdot, \tau)\right\|_{L^{q}}^{p} \mathrm{~d} \tau\right) .
$$


In particular, if $T_{\max }<+\infty$, then

$$
\int_{0}^{T_{\max }}\left\|\lambda_{2}^{+}(\cdot, t)\right\|_{L^{q}}^{p} \mathrm{~d} t=+\infty .
$$

This regularity criterion gives a geometric characterization of the structure of potential finite-time blowup solutions of the Navier-Stokes equation. Theorem 1.4 says that in order for blowup to occur, the flow needs to be stretching in two directions, while compressing more strongly in the third. Points where the strain has two positive eigenvalues, corresponding to stretching in two directions, and one negative eigenvalue, corresponding to compressing more strongly in a third, are the points that drive enstrophy growth and hence blowup. This provides more insight into the qualitative properties of blowup solutions than the regularity criteria that just involve the size of $u$ or $\omega$. The author also proved the following corollary of Theorem 1.4 in 17 .

Corollary 1.5. Suppose $u \in C\left(\left[0, T_{\max }\right) ; \dot{H}^{1}\left(\mathbb{R}^{3}\right)\right)$ is a mild solution of the Navier-Stokes equation. Let $v \in L^{\infty}\left(\mathbb{R}^{3} \times\left[0, T_{\max }\right] ; \mathbb{R}^{3}\right)$, with $|v(x, t)|=1$ almost everywhere. Then for all $\frac{3}{q}+\frac{2}{p}=2, \frac{3}{2}<q \leq+\infty$, there exists $C_{q}>0$ depending only on $q$ such that for all $0<t<T_{\max }$

$$
\|u(\cdot, t)\|_{\dot{H}^{1}}^{2} \leq\left\|u^{0}\right\|_{\dot{H}^{1}}^{2} \exp \left(C_{q} \int_{0}^{t}\|S(\cdot, \tau) v(\cdot, \tau)\|_{L^{q}}^{p} \mathrm{~d} \tau\right) .
$$

In particular, if $T_{\max }<+\infty$, then

$$
\int_{0}^{T_{\max }}\|S(\cdot, t) v(\cdot, t)\|_{L^{q}\left(\mathbb{R}^{3}\right)}^{p} \mathrm{~d} t=+\infty .
$$

This result follows from Theorem 1.4 because $\operatorname{tr}(S)=0$, so $\lambda_{2}$ is the smallest eigenvalue of $S$ in magnitude. Therefore, if $v \in \mathbb{R}^{3},|v|=1$, then

$$
|S v| \geq\left|\lambda_{2}\right|
$$

Corollary 1.5 follows immediately from this fact and Theorem 1.4, We will show in Section 3 that the special case of Corollary 1.5 where we take $v \in \mathbb{R}^{3}$ to be a fixed unit vector is equivalent to Chae and Choe's result, Theorem 1.2 .

This raises an interesting question: can Chae and Choe's result be extended to a regularity criterion on $v \times \omega$, where $v$ is a unit vector that is allowed to vary in space. Clearly, unlike in Corollary [1.5, we won't be able to take an arbitrary unit vector, otherwise we would simply take $v=\frac{\omega}{|\omega|}$, and regularity would be guaranteed for any solution of the Navier-Stokes equation. In fact, using Corollary 1.5, it is possible to improve Chae and Choe's result to one that allows the unit vector $v$ to vary in space and time, so long as $\nabla v$ remains bounded. The main theorem of this paper is the following.

Theorem 1.6. Suppose $u \in C\left(\left[0, T_{\max }\right) ; H^{1}\left(\mathbb{R}^{3}\right)\right)$ is a mild solution of the NavierStokes equation. Suppose $v \in L^{\infty}\left(\mathbb{R}^{3} \times[0,+\infty) ; \mathbb{R}^{3}\right)$, with $|v(x, t)|=1$ almost 
everywhere, and suppose $\nabla v \in L_{\text {loc }}^{\infty}\left([0,+\infty) ; L^{\infty}\left(\mathbb{R}^{3}\right)\right)$. Then for all for all $0<$ $t<T_{\max }$

$$
\begin{aligned}
\|u(\cdot, t)\|_{\dot{H}^{1}}^{2} \leq\left\|u^{0}\right\|_{\dot{H}^{1}}^{2} \exp \left(2916 C_{2}\left\|u^{0}\right\|_{L^{2}}^{4}\|\nabla v\|_{L_{t}^{\infty} L_{x}^{\infty}}^{2}\right. & \\
& \left.+\frac{C_{2}}{8} \int_{0}^{t}\|v(\cdot, \tau) \times \omega(\cdot, \tau)\|_{L^{2}}^{4} \mathrm{~d} \tau\right),
\end{aligned}
$$

where $C_{2}$ is a constant independent of $u$, taken as in Theorem 1.4 and Corollary 1.5.

In particular, if $T_{\max }<+\infty$, then

$$
\int_{0}^{T_{\max }}\|v(\cdot, t) \times \omega(\cdot, t)\|_{L^{2}}^{4} \mathrm{~d} t=+\infty .
$$

Chae and Choe's result, Theorem [1.2, states that for a solution of the NavierStokes equation to blowup in finite-time, the vorticity must become unbounded in every fixed plane. For a two dimensional flow in the $x y$ plane, the vorticity is entirely in the $z$ direction, so Theorem 1.2 can be interpreted as requiring the blowup of vorticity to be globally three dimensional. Theorem [1.6, strengthens this result, by requiring the vorticity to blowup in every plane, where the plane may vary in space and time so long as the gradient of the vector orthogonal to the plane remains bounded, meaning the geometry of the blowup must be locally three dimensional. In particular, this means that in order to blowup in finite-time, a solution of the Navier-Stokes equation must be fully three dimensional at all length scales, which is consistent with the Kolmogorov phenomenological theory of turbulence, as we will discuss shortly.

This result highlights the strength of the strain formulation of the Navier-Stokes regularity problem. Using the strain formulation, we are able to get a stronger regularity criterion in terms of the vorticity than by working directly with the vorticity formulation. Theorem 1.6 follows as a fairly direct corollary of Corollary [1.5 using the relationship between the structure of the strain and vorticity that is imposed by the divergence free condition on the velocity. Because the regularity criterion on $\lambda_{2}^{+}$gives us geometric information that is fundamentally anisotropic in that it does not involve any fixed direction, it allows us to go from a component reduction regularity criterion that involves some fixed direction, and therefore guarantees regularity as long as the solution is not too far away from being globally two dimensional in some sense, to a component reduction type regularity criterion that only requires that the solution is not too far away from being locally two dimensional. The regularity criterion in terms of $\lambda_{2}^{+}$first proven by Neustupa and Penel therefore encodes fundamentally anisotropic information about the structure of possible blowup solutions that does not require the imposition of any arbitrary direction. For this reason it is quite powerful.

We should also note that while Chae and Choe's result holds for $\frac{2}{p}+\frac{3}{q}=2, \frac{3}{2}<$ $q<+\infty$, the proof of Theorem 1.6 only holds with $q=2, p=4$, because the Hilbert space structure of $L^{2}$ is essential to the proof. It is possible that Theorem 1.6 can be generalized for all $\frac{3}{2}<q<+\infty$, but this would require much more delicate analysis, and likely somewhat different techniques.

There are several other scale critical, component reduction type regularity criteria. For instance, Kukavica and Ziane [12] showed that if a smooth solution of the Navier-Stokes equation blows up in finite-time $T_{\max }<+\infty$, and if $\frac{2}{p}+\frac{3}{q}=2$, with 
$\frac{9}{4} \leq q \leq 3$, then

$$
\int_{0}^{T_{\max }}\left\|\partial_{3} u(\cdot, t)\right\|_{L^{q}}^{p} \mathrm{~d} t=+\infty .
$$

More recently, it was shown by Chemin and Zhang [3] and Chemin, Zhang, and Zhang [4 that if $T_{\max }<+\infty$ and $4<p<+\infty$, then

$$
\int_{0}^{T_{\max }}\left\|u_{3}(\cdot, t)\right\|_{\dot{H}^{\frac{1}{2}+\frac{2}{p}}}^{p} \mathrm{~d} t=+\infty .
$$

Neustupa, Novotný, and Penel had previously proven a regularity criterion on $u_{3}$ [18], which was the first result of this type, but their regularity criteria was not scale critical; it required $u_{3}$ to be in the subcritical space $L_{t}^{p} L_{x}^{q}$, with $\frac{2}{p}+\frac{3}{q}=\frac{1}{2}$. Like Theorem 1.2 due to Chae and Choe, all of these results say that the flow must be regular as long as it is not too three dimensional in the sense that either $u_{3}$ or $\partial_{3} u$ remains bounded in the appropriate scale critical space. The rotational invariance of the Navier-Stokes equation implies that there is nothing special about the particular direction $e_{3}$, so $\partial_{3} u$ and $u_{3}$ can be replaced by $v \cdot \nabla u$ and $u \cdot v$ respectively for any unit vector $v \in \mathbb{R}^{3}$, but the vector $v$ cannot be allowed to vary in space. These regularity criteria are therefore global anistotropic regularity criteria in that they require solutions which are globally anisotropic in some sense to remain smooth. Theorem [1.6] is significantly stronger because it is a locally anisotropic regularity criterion which only requires solutions to be locally anisotropic to remain smooth.

There is one recent regularity result that does involve local anisotropy. Kukavica, Rusin and Ziane proved that if $u$ is a suitable weak solution on $\mathbb{R}^{3}$, and for some domain $D \subset \mathbb{R}^{3} \times \mathbb{R}^{+}$, we have $\partial_{3} u \in L_{t}^{p} L_{x}^{q}(D)$, with $\frac{2}{p}+\frac{3}{q}=2, \frac{9}{4} \leq q \leq \frac{5}{2}$, then $u$ is Hölder continuous on $D$ [11. This is a locally anisotropic regularity criterion, because we only require control on $\partial_{3} u$ in some domain, not globally. This implies that the control on the anisotropy is local. This differs from Theorem 1.6. which involves an estimate over the whole space, and is therefore a global, not local regularity criterion, but a global regularity criterion that is locally anisotropic, on account of the direction being allowed to vary. We will discuss the relationship between Kukavica, Rusin, and Ziane's regularity criterion and Theorem 1.6 further in section 3 .

Another regularity criterion related to Theorem [1.6 is the regularity criterion in terms of the vorticity direction proven by Constantin and Fefferman, which states that the direction of the vorticity must vary rapidly in regions where the vorticity is large in order for a solution of the Navier-Stokes equation to blowup [5]. This was a very important advance in that the rapid change of the vorticity direction in regions of large vorticity has long been at least heuristically understood as a fundamental property of turbulent flow, and Constantin and Fefferman's result shows that any Navier-Stokes blowup solution must be "turbulent" in this sense. Indeed, even Leonardo da Vinci's qualitative studies of turbulence in the early 1500s (see Figure 1) show an understanding of the fundamental character of a rapid change in the orientation of vortices in turbulent regions of the fluid to the phenomenon of turbulence [9].

Kolmogorov would rigourously describe the phenomenon depicted purely heuristically by da Vinci with his celebrated theory of turbulent cascades [10, which remains central to our understanding of turbulence. Kolmogorov showed that turbulence should involve a transfer of energy from long length scales to shorter 


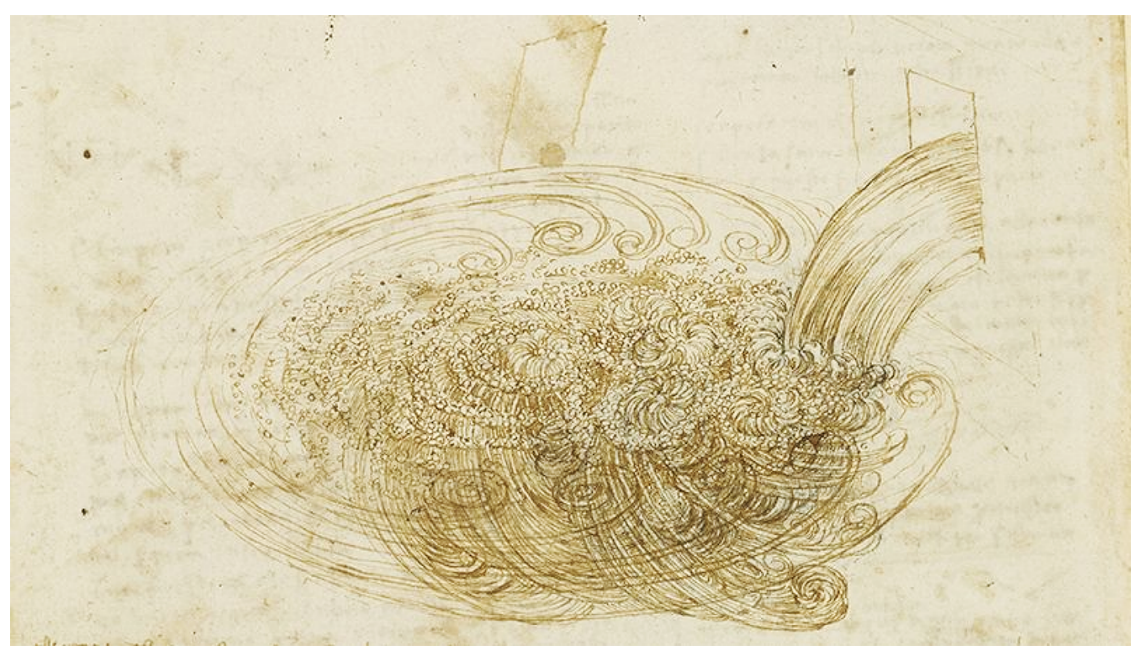

Figure 1. Leonardo da Vinci, Studies of Turbulent Water. Image credit: Royal Collection Trust/C) Her Majesty Queen Elizabeth II 2021.

length scales, giving the appropriate scaling, and also that turbulence is locally isotropic, with no preferred vorticity direction down to the smallest length scales. Kolmogorov's scaling law was extended to Fourier space by Obukhov in [22], where he showed that turbulence should involve a scaling of the energy spectrum of the form $E(\xi) \sim|\xi|^{-\frac{5}{3}}$, in the inertial range.

Constantin and Fefferman were the first to connect the change in the orientation of vortices in turbulent regions to the Navier-Stokes regularity problem, which suggests that blowup for the Navier-Stokes equation is not only of purely mathematical interest, but could have significant physical implications-particularly if there is finite-time blowup - on our phenomenological understanding of turbulence. Constantin and Fefferman's result was later generalized by Beirão da Veiga and Berselli in [1]. In particular, as a corollary of their refined result that the vorticity direction must vary rapidly in regions with large vorticity, they proved a regularity criterion in terms of the gradient of the vorticity direction, $\nabla \frac{\omega}{|\omega|}$.

Theorem 1.7. Suppose $u \in C\left(\left[0, T_{\max }\right) ; H^{1}\right)$ is a mild solution of the NavierStokes equation that blows up in finite-time $T_{\max }<+\infty$. Then for all $\frac{2}{p}+\frac{3}{q}=$ $\frac{1}{2}, 6 \leq q \leq+\infty$.

$$
\int_{0}^{T_{\max }}\left\|\nabla\left(\frac{\omega}{|\omega|}\right)(\cdot, t)\right\|_{L^{q}}^{p} \mathrm{~d} t=+\infty .
$$

This is related to Theorem [1.6, because if we take $v=\frac{\omega}{|\omega|}$, then we find that Theorem 1.6 implies if $T_{\max }<+\infty$, then

$$
\operatorname{ess} \sup _{0<t<T_{\max }}\left\|\nabla\left(\frac{\omega}{|\omega|}\right)(\cdot, t)\right\|_{L^{\infty}}=+\infty .
$$


If we take the $p=4, q=+\infty$ case of Theorem 1.7 then we can see that if a solution of the Navier-Stokes equation blows up in finite-time $T_{\max }<+\infty$, then

$$
\int_{0}^{T_{\max }}\left\|\nabla\left(\frac{\omega}{|\omega|}\right)(\cdot, t)\right\|_{L^{\infty}}^{4} \mathrm{~d} t=+\infty .
$$

We will note that Beirão da Veiga and Berselli's result is stronger than the special case of Theorem 1.6 where we take $v=\frac{\omega}{|\omega|}$ because it not only specifies that $\nabla \frac{\omega}{|\omega|}$, must become unbounded as we approach blowup time, but also a rate of blowup - namely that its integral to the fourth power must go to infinity. We can see that the two extremal cases of Theorem [1.6] are the case where $v$ is constant and hence $\nabla v=0$, in which case we recover Theorem 1.2 from Chae and Choe with $p=4, q=2$, and the case where $v=\frac{\omega}{|\omega|}$ and hence $v \times \omega=0$, in which case we recover a weaker form of Theorem 1.7, from Beirão da Veiga and Berselli. Therefore this result could be said to interpolate between these two results, although suboptimally at one end. We will discuss why this interpolation is suboptimal in section 3 .

In section 2, we will prove the main result of the paper, Theorem 1.6. In section 3 , we will further discuss the relationship between this result and the previous literature.

\section{Proof of the MAIN THEOREM}

We will now prove Theorem 1.6, which is restated here for the reader's convenience.

Theorem 2.1. Suppose $u \in C\left(\left[0, T_{\max }\right) ; H^{1}\left(\mathbb{R}^{3}\right)\right)$ is a mild solution of the NavierStokes equation. Suppose $v \in L^{\infty}\left(\mathbb{R}^{3} \times[0,+\infty) ; \mathbb{R}^{3}\right)$, with $|v(x, t)|=1$ almost everywhere, and suppose $\nabla v \in L_{\text {loc }}^{\infty}\left([0,+\infty) ; L^{\infty}\left(\mathbb{R}^{3}\right)\right)$. Then for all $0<t<T_{\max }$

$$
\begin{aligned}
\|u(\cdot, t)\|_{\dot{H}^{1}}^{2} \leq\left\|u^{0}\right\|_{\dot{H}^{1}}^{2} \exp \left(2916 C_{2}\left\|u^{0}\right\|_{L^{2}}^{4}\|\nabla v\|_{L_{t}^{\infty} L_{x}^{\infty}}^{2}\right. & \\
& \left.+\frac{C_{2}}{8} \int_{0}^{t}\|v(\cdot, \tau) \times \omega(\cdot, \tau)\|_{L^{2}}^{4} \mathrm{~d} \tau\right),
\end{aligned}
$$

where $C_{2}$ is taken is in Theorem 1.4 and Corollary 1.5. In particular, if $T_{\max }<$ $+\infty$, then

$$
\int_{0}^{T_{\max }}\|v(\cdot, t) \times \omega(\cdot, t)\|_{L^{2}}^{4} \mathrm{~d} t=+\infty .
$$

Proof. We will prove that this theorem is a corollary of Corollary 1.5. In particular, we will show that

$$
\begin{aligned}
\int_{0}^{t}\|S(\cdot, \tau) v(\cdot, \tau)\|_{L^{2}}^{4} \mathrm{~d} \tau \leq 2916\left\|u^{0}\right\|_{L^{2}}^{4}\|\nabla v\|_{L_{t}^{\infty} L_{x}^{\infty}}^{2} & \\
& +\frac{1}{8} \int_{0}^{t}\|v(\cdot, \tau) \times \omega(\cdot, \tau)\|_{L^{2}}^{4} \mathrm{~d} \tau .
\end{aligned}
$$

First we will recall from (1.6) that

$$
\begin{aligned}
\frac{1}{2} v \times \omega & =A v \\
& =\frac{1}{2}(\nabla u) v-\frac{1}{2}(\nabla u)^{t r} v .
\end{aligned}
$$


Therefore we find that

$$
\frac{1}{4}\|v \times \omega\|_{L^{2}}^{2}=\frac{1}{4}\|(\nabla u) v\|_{L^{2}}^{2}+\frac{1}{4}\left\|(\nabla u)^{t r} v\right\|_{L^{2}}^{2}-\frac{1}{2}\left\langle(\nabla u) v,(\nabla u)^{t r} v\right\rangle .
$$

Likewise we may compute that

$$
S v=\frac{1}{2}(\nabla u) v+\frac{1}{2}(\nabla u)^{t r} v
$$

and therefore

$$
\|S v\|_{L^{2}}^{2}=\frac{1}{4}\|(\nabla u) v\|_{L^{2}}^{2}+\frac{1}{4}\left\|(\nabla u)^{t r} v\right\|_{L^{2}}^{2}+\frac{1}{2}\left\langle(\nabla u) v,(\nabla u)^{t r} v\right\rangle .
$$

Putting together (2.6) and (2.8), we find that

$$
\|S v\|_{L^{2}}^{2}=\frac{1}{4}\|v \times \omega\|_{L^{2}}^{2}+\left\langle(\nabla u) v,(\nabla u)^{t r} v\right\rangle .
$$

We know that for all $a, b \geq 0,(a+b)^{2} \leq 2 a^{2}+2 b^{2}$, so we find that

$$
\|S v\|_{L^{2}}^{4} \leq \frac{1}{8}\|v \times \omega\|_{L^{2}}^{4}+2\left(\left\langle(\nabla u) v,(\nabla u)^{t r} v\right\rangle\right)^{2} .
$$

Now we will need to estimate $\left\langle(\nabla u) v,(\nabla u)^{t r} v\right\rangle$. By definition we have

$$
\left\langle(\nabla u) v,(\nabla u)^{t r} v\right\rangle=\sum_{i, j, k=1}^{3} \int_{\mathbb{R}^{3}} \partial_{i} u_{j} v_{j} \partial_{k} u_{i} v_{k} \mathrm{~d} x .
$$

Integrating by parts with respect to $x_{i}$, and applying the fact that $\nabla \cdot u=\sum_{i=1}^{3} \partial_{i} u_{i}$ $=0$, we find that

$$
\begin{aligned}
\left\langle(\nabla u) v,(\nabla u)^{t r} v\right\rangle & =-\sum_{i, j, k=1}^{3} \int_{\mathbb{R}^{3}} u_{j} \partial_{k} u_{i}\left(v_{k} \partial_{i} v_{j}+v_{j} \partial_{i} v_{k}\right) \mathrm{d} x \\
& \leq \sum_{i, j, k=1}^{3} \int_{\mathbb{R}^{3}} 2|u||\nabla u\|v\| \nabla v| \\
& =54 \int_{\mathbb{R}^{3}}|u\|\nabla u\| \nabla v| \\
& \leq 54\|u\|_{L^{2}}\|\nabla u\|_{L^{2}}\|\nabla v\|_{L^{\infty}} .
\end{aligned}
$$

This estimate implies that

$$
\|S v\|_{L^{2}}^{4} \leq \frac{1}{8}\|v \times \omega\|_{L^{2}}^{4}+5832\|u\|_{L^{2}}^{2}\|\nabla u\|_{L^{2}}^{2}\|\nabla v\|_{L^{\infty}}^{2} .
$$

Applying the energy equality and our hypothesis on $\nabla v$, we find that

$$
\begin{aligned}
& \int_{0}^{t}\|u(\cdot, \tau)\|_{L^{2}}^{2}\|\nabla u(\cdot, \tau)\|_{L^{2}}^{2}\|\nabla v(\cdot, \tau)\|_{L^{\infty}}^{2} \mathrm{~d} \tau \\
& \leq \int_{0}^{t}\left\|u^{0}\right\|_{L^{2}}^{2}\|\nabla u(\cdot, \tau)\|_{L^{2}}^{2}\|\nabla v\|_{L_{t}^{\infty} L_{x}^{\infty}}^{2} \mathrm{~d} \tau \\
& \quad=\left\|u^{0}\right\|_{L^{2}}^{2}\|\nabla v\|_{L_{t}^{\infty} L_{x}^{\infty}}^{2} \int_{0}^{t}\|\nabla u(\cdot, \tau)\|_{L^{2}}^{2} \mathrm{~d} \tau \\
& \quad \leq \frac{1}{2}\left\|u^{0}\right\|_{L^{2}}^{4}\|\nabla v\|_{L_{t}^{\infty} L_{x}^{\infty}}^{2} .
\end{aligned}
$$


Therefore we may conclude that

$$
\begin{aligned}
\int_{0}^{t}\|S(\cdot, \tau) v(\cdot, \tau)\|_{L^{2}}^{4} \mathrm{~d} \tau \leq 2916\left\|u^{0}\right\|_{L^{2}}^{4} & \|\nabla v\|_{L_{t}^{\infty} L_{x}^{\infty}}^{2} \\
& +\frac{1}{8} \int_{0}^{t}\|v(\cdot, \tau) \times \omega(\cdot, \tau)\|_{L^{2}}^{4} \mathrm{~d} \tau
\end{aligned}
$$

Applying Corollary [1.5, this completes the proof.

\section{Relationship to the previous literature}

In this section, we will further discuss the relationship between the results in this paper and the previous literature. In particular, we will show that Theorem 1.5, the regularity criterion on $S v$, is equivalent to Chae and Choe's regularity criterion on $v \times \omega$ in the special case where we take $v \in \mathbb{R}^{3}$ to be a fixed unit vector. In order to do this we will first need to introduce the Helmholtz decomposition of vector fields in $L^{q}, 1<q<+\infty$ into gradients and divergence free vector fields.

Proposition 3.1. Suppose $1<q<+\infty$. For all $v \in L^{q}\left(\mathbb{R}^{3} ; \mathbb{R}^{3}\right)$, there exists a unique $u \in L^{q}\left(\mathbb{R}^{3} ; \mathbb{R}^{3}\right), \nabla \cdot u=0$ and $\nabla f \in L^{q}\left(\mathbb{R}^{3} ; \mathbb{R}^{3}\right)$ such that $v=u+\nabla f$, and there exists $C_{q} \geq 1$ depending only on $q$, such that

$$
\|u\|_{L^{q}} \leq C_{q}\|v\|_{L^{q}}
$$

and

$$
\|\nabla f\|_{L^{q}} \leq C_{q}\|v\|_{L^{q}}
$$

Note because we do not have any assumptions of higher regularity, we will say that $\nabla \cdot u=0$, if for all $\phi \in C_{c}^{\infty}\left(\mathbb{R}^{3}\right)$

$$
\int_{\mathbb{R}^{3}} u \cdot \nabla \phi=0
$$

and we will say that $\nabla f$ is a gradient if for all $w \in C_{c}^{\infty}\left(\mathbb{R}^{3} ; \mathbb{R}^{3}\right), \nabla \cdot w=0$, we have

$$
\int_{\mathbb{R}^{3}} \nabla f \cdot w=0
$$

Using this decomposition, we will define the projection onto the space of divergence free vector fields and space of gradients in $L^{q}$.

Definition 3.2. For all $1<q<+\infty$, let the projection onto the space of divergence free vector fields, $P_{d f}: L^{q} \rightarrow L^{q}$, be given by

$$
P_{d f}(v)=u,
$$

and let the projection onto the space of gradients, $P_{g r}: L^{q} \rightarrow L^{q}$, be given by

$$
P_{g r}(v)=\nabla f
$$

where $v, u, \nabla f$ are taken as in Proposition 3.1. Note in particular that Proposition 3.1 implies that $P_{d f}$ and $P_{g r}$ are bounded operators on $L^{q}$.

We can now show that for any fixed unit vector $v \in \mathbb{R}^{3}\|S v\|_{L^{q}}$ and $\|v \times \omega\|_{L^{q}}$ are equivalent norms. 
Proposition 3.3. Suppose $\nabla \cdot u=0$, and $\nabla u \in L^{q}$ Then for all unit vectors $v \in \mathbb{R}^{3}$, and for all $1<q<+\infty$

$$
\begin{aligned}
& \|(v \cdot \nabla) u\|_{L^{q}} \leq C_{q}\|v \times \omega\|_{L^{q}}, \\
& \|(v \cdot \nabla) u\|_{L^{q}} \leq C_{q}\|2 S v\|_{L^{q}}, \\
& \|\nabla(u \cdot v)\|_{L^{q}} \leq C_{q}\|v \times \omega\|_{L^{q}}, \\
& \|\nabla(u \cdot v)\|_{L^{q}} \leq C_{q}\|2 S v\|_{L^{q}},
\end{aligned}
$$

where $C_{q}$ is taken as in Proposition 3.1. Furthermore,

$$
\frac{1}{2 C_{q}}\|v \times \omega\|_{L^{q}} \leq\|2 S v\|_{L^{q}} \leq 2 C_{q}\|v \times \omega\|_{L^{q}} .
$$

Proof. First we will observe that the rotational invariance of the space of divergence free vector fields means that we can take $v=e_{3}$ without loss of generality. It is a simple calculation to see that

$$
e_{3} \times \omega=\nabla u_{3}-\partial_{3} u .
$$

Likewise we can see that

$$
2 S e_{3}=\nabla u_{3}+\partial_{3} u .
$$

Clearly $\nabla u_{3}$ is a gradient, and we can also see that $\partial_{3} u$ is divergence free because

$$
\begin{aligned}
\nabla \cdot \partial_{3} u & =\partial_{3} \nabla \cdot u \\
& =0 .
\end{aligned}
$$

This implies that

$$
\begin{aligned}
& \partial_{3} u=-P_{d f}\left(e_{3} \times \omega\right), \\
& \partial_{3} u=P_{d f}\left(2 S e_{3}\right) .
\end{aligned}
$$

Applying Proposition 3.1 we can therefore conclude that

$$
\begin{aligned}
\left\|\partial_{3} u\right\|_{L^{q}} & \leq C_{q}\left\|e_{3} \times \omega\right\|_{L^{q}}, \\
\left\|\partial_{3} u\right\|_{L^{q}} & \leq C_{q}\left\|2 S e_{3}\right\|_{L^{q}} .
\end{aligned}
$$

Likewise we can observe that

$$
\begin{aligned}
& \nabla u_{3}=P_{g r}\left(e_{3} \times \omega\right), \\
& \nabla u_{3}=P_{g r}\left(2 S e_{3}\right),
\end{aligned}
$$

and apply Proposition 3.1 to find that

$$
\begin{aligned}
\left\|\nabla u_{3}\right\|_{L^{q}} & \leq C_{q}\left\|e_{3} \times \omega\right\|_{L^{q}}, \\
\left\|\nabla u_{3}\right\|_{L^{q}} & \leq C_{q}\left\|2 S e_{3}\right\|_{L^{q}} .
\end{aligned}
$$

We will now finish the proof by applying the triangle inequality and concluding that

$$
\begin{aligned}
\left\|e_{3} \times \omega\right\|_{L^{q}} & =\left\|\nabla u_{3}-\partial_{3} u\right\|_{L^{q}} \\
& \leq\left\|\nabla u_{3}\right\|_{L^{q}}+\left\|\partial_{3} u\right\|_{L^{q}} \\
& \leq 2 C_{q}\left\|2 S e_{3}\right\|_{L^{q}},
\end{aligned}
$$


and that

$$
\begin{aligned}
\left\|2 S e_{3}\right\|_{L^{q}} & =\left\|\nabla u_{3}+\partial_{3} u\right\|_{L^{q}} \\
& \leq\left\|\nabla u_{3}\right\|_{L^{q}}+\left\|\partial_{3} u\right\|_{L^{q}} \\
& \leq 2 C_{q}\left\|e_{3} \times \omega\right\|_{L^{q}} .
\end{aligned}
$$

This completes the proof.

Proposition 3.3 implies that in the range $\frac{9}{4} \leq q \leq 3$, Kukavica and Ziane's regularity criterion on $\partial_{3} u$ implies Chae and Choe's regularity criterion on $e_{3} \times \omega$ because

$$
\left\|\partial_{3} u\right\|_{L^{q}} \leq C_{q}\left\|e_{3} \times \omega\right\|_{L^{q}} .
$$

Likewise, Chemin, Zhang, and Zhang's regularity criterion on $u_{3}$ implies Chae and Choe's regularity criterion on $e_{3} \times \omega$ when $4<p<+\infty$. We can see this by applying the fractional Sobolev embedding $W^{1, q}\left(\mathbb{R}^{3}\right) \hookrightarrow \dot{H}^{\frac{1}{2}+\frac{2}{p}}\left(\mathbb{R}^{3}\right)$ when $\frac{2}{p}+\frac{3}{q}=2$, and Proposition 3.3 to find

$$
\begin{aligned}
\left\|u_{3}\right\|_{\dot{H}^{\frac{1}{2}+\frac{2}{p}}} & \leq C_{q}\left\|\nabla u_{3}\right\|_{L^{q}} \\
& \leq \tilde{C}_{q}\left\|e_{3} \times \omega\right\|_{L^{q}}
\end{aligned}
$$

We will also note that Theorem 1.6 differs in a fundamental way from the locally anisotropic regularity criterion proven by Kukavica, Rusin, and Ziane in [11. This is true in particular because while

$$
\left\|\partial_{3} u\right\|_{L^{q}\left(\mathbb{R}^{3}\right)} \leq C_{q}\left\|e_{3} \times \omega\right\|_{L^{q}\left(\mathbb{R}^{3}\right)},
$$

no such inequality holds if we are not on the whole space. For arbitrary $\nabla u \in$ $L^{q}\left(\mathbb{R}^{3}\right), \nabla \cdot u=0$, and arbitrary domains $\Omega \subset \mathbb{R}^{3}$, there is no inequality of the form

$$
\left\|\partial_{3} u\right\|_{L^{q}(\Omega)} \leq C_{q}\left\|e_{3} \times \omega\right\|_{L^{q}(\Omega)} .
$$

The proof of Proposition 3.3 relies on the $L^{q}$ boundedness of the Helmholtz decomoposition, which in turn relies on the $L^{q}$ boundedness of the Riesz transform. This is something which only holds globally, not locally, because the Riesz transform is a nonlocal operator. $e_{3} \times \omega$ controls $\partial_{3} u$ globally in $L^{q}$, but not locally in $L^{q}$. This means that while Kukavica and Ziane's regularity criterion for $\partial_{3} u \in L_{t}^{p} L_{x}^{q}\left(\mathbb{R}^{3}\right)$ implies Chae and Choe's regularity criterion for $e_{3} \times \omega \in L_{t}^{p} L_{x}^{q}\left(\mathbb{R}^{3}\right)$ for $\frac{9}{4} \leq q \leq 3$, the local anisotropic regularity criterion with $\partial_{3} u \in L_{t}^{p} L_{x}^{q}(D)$ and the locally anisotropic regularity criterion on $v \times \omega$, where $v$ is allowed to vary do not have this same relationship.

Finally, we will note that when we set $v=\frac{\omega}{|\omega|}$, Theorem 1.6 requires that if our solution of the Navier-Stokes equation blows up in finite-time $T_{\max }<+\infty$, then

$$
{\operatorname{ess} \sup _{(x, t) \in \mathbb{R}^{3} \times\left[0, T_{\max }\right)}}\left|\nabla\left(\frac{\omega}{|\omega|}\right)(x, t)\right|=+\infty,
$$

as long as $\omega=0$ on at most a set of measure zero. However, as we discussed in the introduction, Beirão da Veiga and Berselli proved the stronger result that under these conditions

$$
\int_{0}^{T_{\max }}\left\|\nabla\left(\frac{\omega}{|\omega|}\right)(\cdot, t)\right\|_{L^{\infty}}^{4} \mathrm{~d} t=+\infty .
$$


For this reason, it seems like it ought to be possible to relax the condition in Theorem 1.6 from $\nabla v \in L_{t}^{\infty} L_{x}^{\infty}$ to $\nabla v \in L_{t}^{4} L_{x}^{\infty}$. The methods used to prove Theorem 1.6 would at first glance suggest this would be possible, but the difficulty is that when integrating by parts, we cannot get all of the derivatives off of $u$ and onto $v$, which is what leads to the sub-optimal result in this endpoint case.

Remark 3.4. Suppose for all $u \in H^{1}, \nabla \cdot u=0, v \in L^{\infty},|v(x)|=1$ almost everywhere $x \in \mathbb{R}^{3}$, we had a bound of the form

$$
\begin{aligned}
\left|\left\langle(\nabla u) v,(\nabla u)^{t r} v\right\rangle\right| & \leq C \int_{\mathbb{R}^{3}}|u|^{2}|\nabla v|^{2} \\
& \leq C\|u\|_{L^{2}}^{2}\|\nabla v\|_{L^{\infty}}^{2} .
\end{aligned}
$$

Then using the energy inequality we could conclude that

$$
\begin{aligned}
\int_{0}^{t}\left|\left\langle(\nabla u) v,(\nabla u)^{t r} v\right\rangle(\tau)\right|^{2} \mathrm{~d} \tau & \leq C^{2} \int_{0}^{t}\|u(\cdot, \tau)\|_{L^{2}}^{4}\|\nabla v(\cdot, \tau)\|_{L^{\infty}}^{4} \mathrm{~d} \tau \\
& \leq C^{2}\left\|u^{0}\right\|_{L^{2}}^{4} \int_{0}^{t}\|\nabla v(\cdot, \tau)\|_{L^{\infty}}^{4} \mathrm{~d} \tau
\end{aligned}
$$

and we could relax the requirement in Theorem 1.6 to $\nabla v \in L_{t}^{4} L_{x}^{\infty}$ using precisely the same proof. The difficulty is that, as much as at first glance it would appear that an inequality of the form (3.37) should hold using integration by parts, after multiple attempts to do so by the author it does not appear possible to push all the derivatives off of $u$ and onto $v$ in the manner desired. The asymmetry from the transpose, $(\nabla u)^{t r}$, does not seem to allow this. It is definitely possible, perhaps even likely, that the condition can be relaxed to $\nabla v \in L_{t}^{4} L_{x}^{\infty}$, which would make the interpolation between Chae and Choe's regularity criterion and Beirão da Veiga and Berselli's optimal, but the proof would probably require some fundamentally new ideas, because it is unlikely to follow as a corollary of the regularity criterion on $\lambda_{2}^{+}$in Theorem 1.4

\section{REFERENCES}

[1] Hugo Beirão da Veiga and Luigi C. Berselli, On the regularizing effect of the vorticity direction in incompressible viscous flows, Differential Integral Equations 15 (2002), no. 3, 345-356. MR.1870646

[2] Dongho Chae and Hi-Jun Choe, Regularity of solutions to the Navier-Stokes equation, Electron. J. Differential Equations (1999), No. 05, 7. MR.1673067

[3] Jean-Yves Chemin and Ping Zhang, On the critical one component regularity for 3-D NavierStokes systems (English, with English and French summaries), Ann. Sci. Éc. Norm. Supér. (4) 49 (2016), no. 1, 131-167, DOI 10.24033/asens.2278. MR3465978

[4] Jean-Yves Chemin, Ping Zhang, and Zhifei Zhang, On the critical one component regularity for 3-D Navier-Stokes system: general case, Arch. Ration. Mech. Anal. 224 (2017), no. 3, 871-905, DOI 10.1007/s00205-017-1089-0. MR3621812

[5] Peter Constantin and Charles Fefferman, Direction of vorticity and the problem of global regularity for the Navier-Stokes equations, Indiana Univ. Math. J. 42 (1993), no. 3, 775-789, DOI 10.1512/iumj.1993.42.42034. MR.1254117

[6] L. Iskauriaza, G. A. Serëgin, and V. Shverak, $L_{3, \infty}$-solutions of Navier-Stokes equations and backward uniqueness (Russian, with Russian summary), Uspekhi Mat. Nauk 58 (2003), no. 2(350), 3-44, DOI 10.1070/RM2003v058n02ABEH000609; English transl., Russian Math. Surveys 58 (2003), no. 2, 211-250. MR1992563

[7] Hiroshi Fujita and Tosio Kato, On the Navier-Stokes initial value problem. I, Arch. Rational Mech. Anal. 16 (1964), 269-315, DOI 10.1007/BF00276188. MR.166499 
[8] Zhengguang Guo, Petr Kučera, and Zdeněk Skalák, Regularity criterion for solutions to the Navier-Stokes equations in the whole 3D space based on two vorticity components, J. Math. Anal. Appl. 458 (2018), no. 1, 755-766, DOI 10.1016/j.jmaa.2017.09.029. MR3711930

[9] Martin Kemp, Leonardo da Vinci's laboratory: studies in flow, Nature 571 (2019), 322-323.

[10] A. Kolmogoroff, The local structure of turbulence in incompressible viscous fluid for very large Reynold's numbers, C. R. (Doklady) Acad. Sci. URSS (N.S.) 30 (1941), 301-305. MR0004146

[11] Igor Kukavica, Walter Rusin, and Mohammed Ziane, Localized anisotropic regularity conditions for the Navier-Stokes equations, J. Nonlinear Sci. 27 (2017), no. 6, 1725-1742, DOI 10.1007/s00332-017-9382-5. MR 3713929

[12] Igor Kukavica and Mohammed Ziane, Navier-Stokes equations with regularity in one direction, J. Math. Phys. 48 (2007), no. 6, 065203, 10, DOI 10.1063/1.2395919. MR.2337002

[13] O. A. Ladyženskaja, Uniqueness and smoothness of generalized solutions of Navier-Stokes equations (Russian), Zap. Naučn. Sem. Leningrad. Otdel. Mat. Inst. Steklov. (LOMI) 5 (1967), 169-185. MR0236541

[14] Pierre Gilles Lemarié-Rieusset, The Navier-Stokes problem in the 21st century, CRC Press, Boca Raton, FL, 2016. MR 3469428

[15] Jean Leray, Sur le mouvement d'un liquide visqueux emplissant l'espace (French), Acta Math. 63 (1934), no. 1, 193-248, DOI 10.1007/BF02547354. MR.1555394

[16] Andrew J. Majda and Andrea L. Bertozzi, Vorticity and incompressible flow, Cambridge Texts in Applied Mathematics, vol. 27, Cambridge University Press, Cambridge, 2002. MR:1867882

[17] Evan Miller, A regularity criterion for the Navier-Stokes equation involving only the middle eigenvalue of the strain tensor, Arch. Ration. Mech. Anal. 235 (2020), no. 1, 99-139, DOI 10.1007/s00205-019-01419-z. MR 4062474

[18] Jirí Neustupa, Antonín Novotný, and Patrick Penel, An interior regularity of a weak solution to the Navier-Stokes equations in dependence on one component of velocity, Topics in mathematical fluid mechanics, Quad. Mat., vol. 10, Dept. Math., Seconda Univ. Napoli, Caserta, 2002, pp. 163-183. MR2051774

[19] Jiří Neustupa and Patrick Penel, Anisotropic and geometric criteria for interior regularity of weak solutions to the 3D Navier-Stokes equations, Mathematical fluid mechanics, Adv. Math. Fluid Mech., Birkhäuser, Basel, 2001, pp. 237-265. MR.1865056

[20] Jiř́i Neustupa and Patrick Penel, The role of eigenvalues and eigenvectors of the symmetrized gradient of velocity in the theory of the Navier-Stokes equations (English, with English and French summaries), C. R. Math. Acad. Sci. Paris 336 (2003), no. 10, 805-810, DOI 10.1016/S1631-073X(03)00174-2. MR1990019

[21] Jiří Neustupa and Patrick Penel, Regularity of a weak solution to the Navier-Stokes equation in dependence on eigenvalues and eigenvectors of the rate of deformation tensor, Trends in partial differential equations of mathematical physics, Progr. Nonlinear Differential Equations Appl., vol. 61, Birkhäuser, Basel, 2005, pp. 197-212, DOI 10.1007/3-7643-7317-2_15. MR.2129619

[22] A. Obukhoff, On the energy distribution in the spectrum of a turbulent flow, C. R. (Doklady) Acad. Sci. URSS (N.S.) 32 (1941), 19-21. MR0005852

[23] Giovanni Prodi, Un teorema di unicità per le equazioni di Navier-Stokes (Italian), Ann. Mat. Pura Appl. (4) 48 (1959), 173-182, DOI 10.1007/BF02410664. MR126088

[24] James Serrin, On the interior regularity of weak solutions of the Navier-Stokes equations, Arch. Rational Mech. Anal. 9 (1962), 187-195, DOI 10.1007/BF00253344. MR 136885

[25] Zhang Zhifei and Chen Qionglei, Regularity criterion via two components of vorticity on weak solutions to the Navier-Stokes equations in $\mathbb{R}^{3}$, J. Differential Equations 216 (2005), no. 2, 470-481, DOI 10.1016/j.jde.2005.06.001. MR2165305

Department of Mathematics and Statistics, McMaster University, Hamilton, OnTARIO, CANADA

Email address: emiller@msri.org 